\title{
Seismic Anisotropy of the Upper Crust in the Mountain Ranges of Taiwan from the TAIGER Explosion Experiment
}

\author{
Hao Kuo-Chen ${ }^{1, *}$, Piotr Środa ${ }^{2}$, Francis $\mathrm{Wu}^{1,3,4}$, Chien-Ying Wang ${ }^{1}$, and Yao-Wen Kuo ${ }^{1}$ \\ ${ }^{1}$ Department of Earth Sciences, National Central University, Jhongli, Taiwan \\ ${ }^{2}$ Institute of Geophysics, Polish Academy of Sciences, Warsaw, Poland \\ ${ }^{3}$ Department of Geological and Environmental Sciences, State University of New York at Binghamton, Binghamton, USA \\ ${ }^{4}$ Department of Earth Sciences, University of Southern California, Los Angeles, USA
}

Received 18 March 2013, accepted 30 July 2013

\begin{abstract}
Taiwan is known as a strongly anisotropic region observed from SKS 1 - 2 s delay time and other teleseismic phases. An estimate of the crustal contribution to the total anisotropy from the foliated Central Range is essential to understanding the overall teleseismic results. We used $\mathrm{P}$ wave arrivals from the dense seismic arrays deployed during the TAIGER active source experiments and the permanent broadband seismic stations to determine the crustal anisotropy. From the arrival time analysis as a function of azimuth, we detected a clear $\cos (2 \theta)$ pattern. The strength of the crustal anisotropy $(0-15 \mathrm{~km}$ depth $)$ reaches $8-10 \%$ and the fast direction azimuth is around $35-43^{\circ}$ for the overall mountain ranges. The anisotropic variations from the central to the north are found in detail. The results could indicate that the upper crustal delay time contribution of teleseismic shear waves reaches up to $0.45 \mathrm{~s}$. The geological data at the surface and geophysical observations imply a coherent deformation from the surface to the lower crust or even down to the upper mantle.
\end{abstract}

Key words: Seismic anisotropy, Foliation, Taiwan orogeny

Citation: Kuo-Chen, H., P. Sroda, F. Wu, C. Y. Wang, and Y. W. Kuo, 2013: Seismic anisotropy of the upper crust in the mountain ranges of Taiwan from the TAIGER explosion experiment. Terr. Atmos. Ocean. Sci., 24, 963-970, doi: 10.3319/TAO.2013.07.30.01(T)

\section{INTRODUCTION}

Young Taiwan orogeny ( 6.5 Ma; Chai 1972) is bracketed by two subduction systems: the Eurasian plate subducts eastward under the Philippine sea plate along the Manila trench in the south, whereas, the Philippine sea plate subducts northwestward beneath the Eurasian plate along the Ryukyu trench in the north (Fig. 1). The Longitudinal Valley Fault (LVF) as a suture zone separates these two plates. In the mountain ranges, the Central Range (CR) is formed by the pre-Tertiary basement of the continental margin and the Miocene to Eocene slates and the Hsuehshan Range (HR) is composed of the Eocene and Oligocene continental shelf sediments. These two ranges (CR and HR) are metamorphic rocks exposed at the surface with a general NNE direction of foliation (green segments of Fig. 1) (Ho 1988). In the mountain belts, the intensively deformed crust is disclosed by a thickened crustal root (e.g., Kuo-Chen et al. 2012) with

\footnotetext{
* Corresponding author

E-mail:kuochen@ncu.edu.tw
}

a rapid uplift rate of $\sim 20 \mathrm{~mm} \mathrm{yr}^{-1}$ at the surface (Ching et al. 2011).

Teleseismic S wave splitting measurement studies show that the fast directions are parallel to the foliation and structural trends of the mountain belts with $1-2$ s delay time (Rau et al. 2000; Huang et al. 2006; Kuo-Chen et al. 2009). The results indicate the crust and upper mantle deformed coherently at least down to $100 \mathrm{~km}$ depth if we assume an averaged 4\% anisotropy, as proposed by Silver (1996). Although from the rock measurements of the slate belt in the Central Range, those rocks could be highly anisotropic, as high as $15 \%$ for $\mathrm{P}$ wave (Wu et al. 2007). Studies of local shear wave splitting measurements in the southern Central Range have recorded 0 to $0.3 \mathrm{~s}$ delay time, but mostly less than $0.1 \mathrm{sec}$ for paths mostly in the southern Central Range. These delay times correspond to $0-6 \%$ anisotropy in the upper $30 \mathrm{~km}$ of the crust (Chang et al. 2009). However, the rock property from local shear wave splitting measurements was obtained at sparse sites, which cannot represent the anisotropy of the crustal scale in whole mountain 


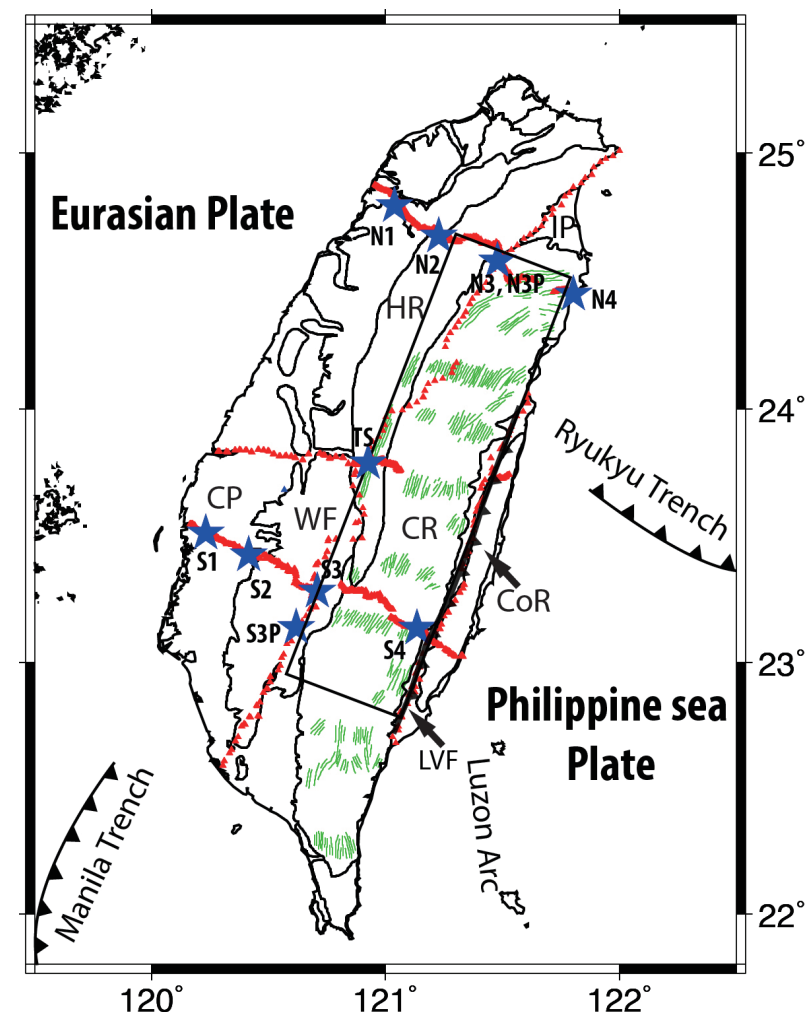

Fig. 1. Tectonic setting of Taiwan. CP: Coastal Plain; WF: Western Foothills; HR: Hsuehshan Range; CR: Central Range; CoR: Coastal Range; IP: Ilan Plain; LVF: Longitudinal Valley Fault. Green segments: foliated trends of the Central Range. Red triangles: seismic station. Blue stars: shot locations. Rectangular area: study region.

ranges. Because of the lack of vertical resolution from the teleseismic shear wave splitting results, the crustal anisotropy estimate is critical for obtaining a reliable estimate of the anisotropy in the upper mantle. Sroda (2006) reported the upper crustal anisotropy based on the $\mathrm{P}$ wave arrivals from an active source experiment in the mountain ranges of southeastern Poland that can provide the overall magnitude and pattern of anisotropy in the crust. In 2006 and 2008 the TAIGER (Taiwan Integrated Geodynamic Research) project conducted 11 explosions and large amount of dense seismic arrays deployed along two EW and NS transects across the mountain ranges (Fig. 1). This data offered a good opportunity to map the crustal anisotropy from the $\mathrm{P}$ wave in the Central Taiwan Range for the first time.

\section{DATA}

The TAIGER project executed 10 explosions in 2008 along two EW transects (southern and northern lines) with two NS transects and two small patches of EW arrays in central Taiwan to study the crustal structures. One-component, $4.5 \mathrm{~Hz}$ geophones, were deployed along the EW transects with $200 \mathrm{~m}$ spacing. Four hundred fifty-five and 609 stations were deployed along the northern and southern lines, respectively. Fifty-six and 109 stations were deployed in the east and west of the NS transects with $2 \mathrm{~km}$ spacing, respectively (Fig. 1). The permanent broadband stations in the mountain ranges are also included in this study (blue triangles, Fig. 2a). The southern and northern lines recorded only 5 in-line shots for imaging 2D crustal structures. However, two NS transects recorded 10 shots, including the off-line shots. Besides those 10 shots, we also include the records from 1 shot in 2006 in central Taiwan (Fig. 1). With those four transects we can analyze the crustal anisotropy of $\mathrm{P}$ wave arrivals with wide azimuthal coverage of the ray paths across the mountain ranges.

Eight shots and 1293 of Pg recordings in the $30-150 \mathrm{~km}$ offset range within the mountain belts are used (Fig. 2a). A minimum of $30 \mathrm{~km}$ and a maximum of $150 \mathrm{~km}$ offset are applied to exclude the local-scale effects (low velocity layer) and Pn arrivals, respectively (Figs. 2b and c). In Fig. 3, from the travel time diagram of the refracted arrivals, we are able to see a dependence on azimuth with a trend of $\cos (2 \theta)$ form. The amplitude of the periodic trend is significantly larger than the travel time variations for the velocity inhomogeneities. The travel time minimum is at about $30^{\circ}$, which is subparallel to the structural trend of the Central Range.

\section{ANISOTROPIC DELAY-TIME INVERSION}

Several algorithms for seismic active source data anisotropic tomography were recently developed that allow detailed 3-D model parameterization. However, the data set used in this study was obtained in an experiment oriented to record mainly 2-D seismic sections and was not focused on obtaining full azimuthal coverage with high fold for each receiver. Therefore, we limited this study to obtaining the most robust and reliable result assuming a simple model of the medium for the travel time inversion of the delay-time method anisotropic variant. In this algorithm, originally presented by Willmore and Bancroft (1960), the medium consists of an upper layer with varying velocity and thickness and a lower layer of unknown, constant, isotropic velocity $\mathrm{V}$. The modification of this method for an anisotropic lower layer [used e.g., by Song et al. (2001)] assumes weak azimuthal slowness variability according to the Backus (1965) formula. The resulting expression for the travel time of a refracted ray has the form:

$$
\begin{aligned}
t_{i j}= & a_{i}+b_{j}+D_{i j}\left[S_{0}+A \cos (2 \theta)+B \sin (2 \theta)+\right. \\
& C \cos (4 \theta)+D \sin (4 \theta)]
\end{aligned}
$$

where $D_{i j}$ is the distance from the i-th source to the $j$-th receiver, $S_{0}$ is unknown $P$ wave slowness $(1 / \mathrm{V})$ below the refractor, $a_{i}, b_{j}$ are unknown time delays for the $i$-th source (source corrections) and the $\mathrm{j}$-th receiver (receiver corrections), respectively, and $\mathrm{A}, \mathrm{B}, \mathrm{C}$, and $\mathrm{D}$ are small unknown 
(a)

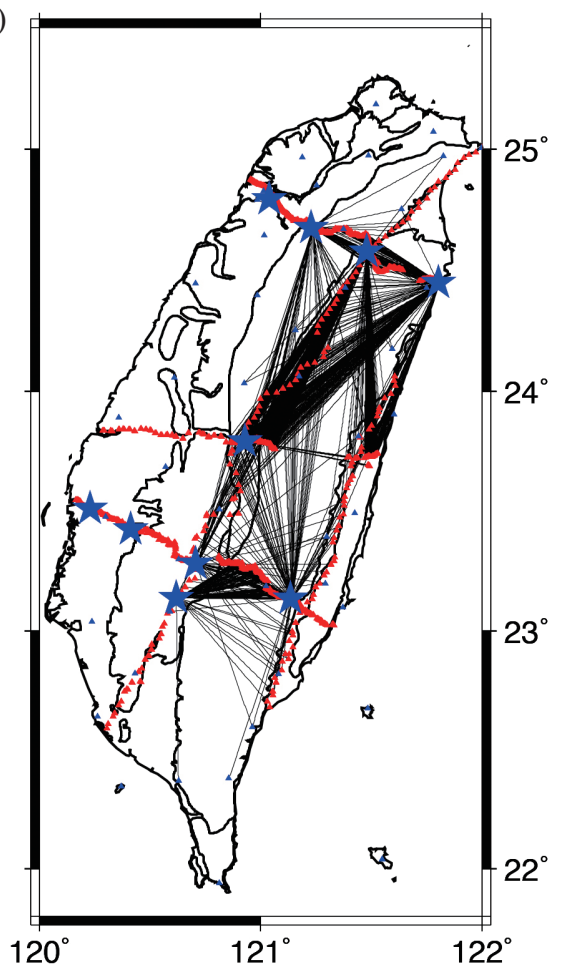

(b)

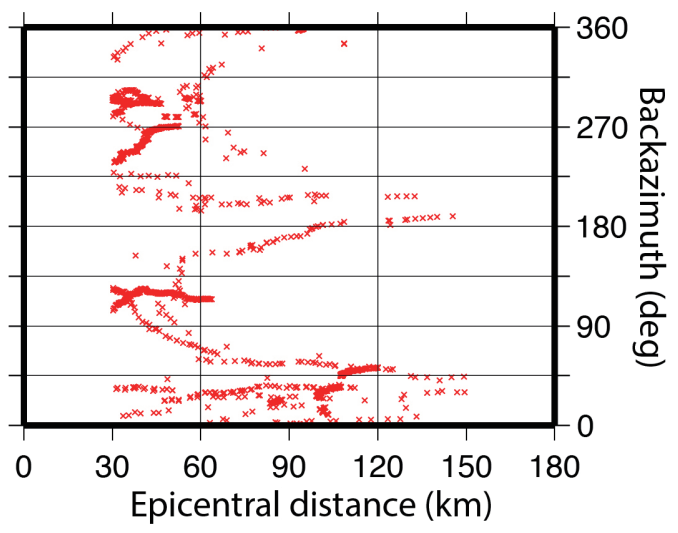

(c)

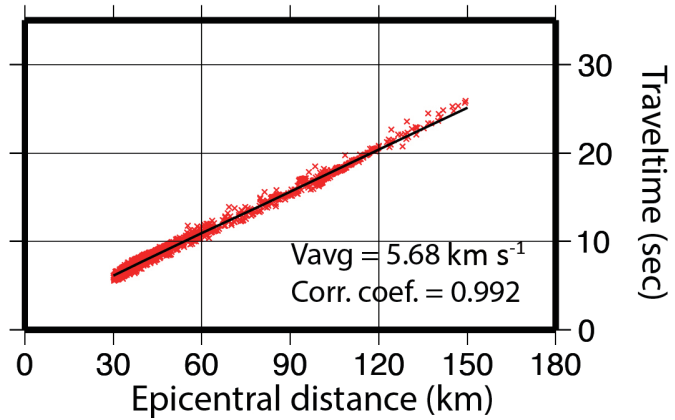

Fig. 2. Ray paths of the selected explosion arrivals (a), back-azimuthal coverage (b), and the mean velocity (c). Black lines: ray paths. Red triangles: seismic stations. Blue stars: shot locations.

(a)

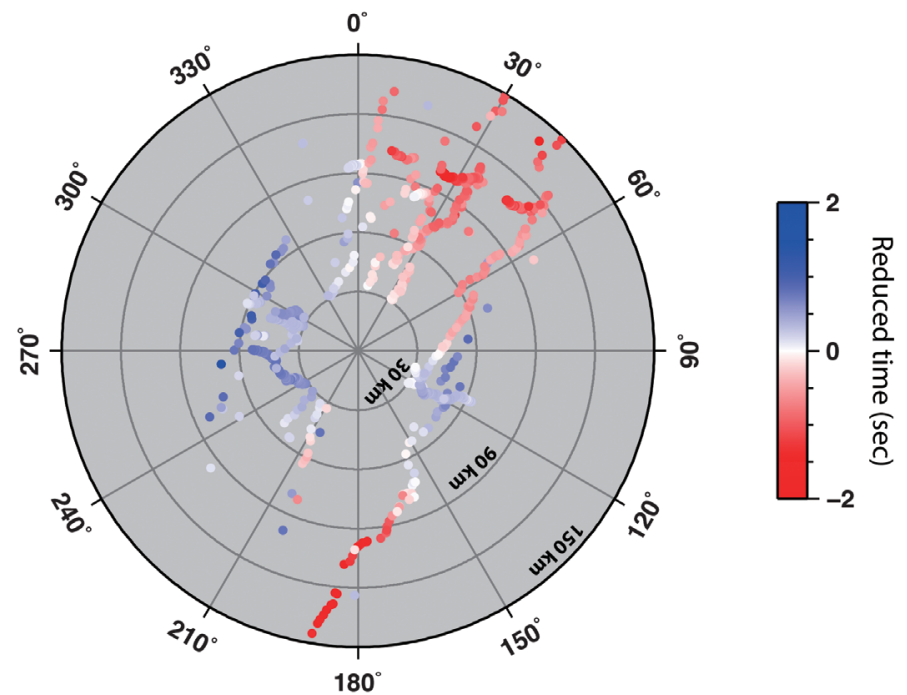

Fig. 3. Azimuthal diagrams of Pg travel times for $30-150 \mathrm{~km}$ offset. (a) Polar diagram of travel times. (b) Azimuthal diagrams of Pg travel times from different shots. Reduced time is $5.6 \mathrm{~km} \mathrm{~s}^{-1}$.

(b)

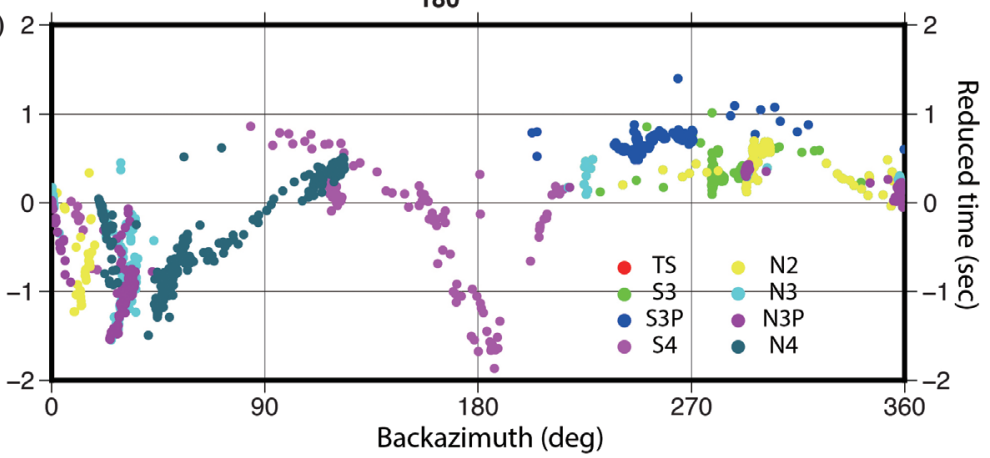


coefficients which are the elastic parameters defining weak anisotropy. Equation (1) is valid for weak anisotropy, i.e., for elastic parameters (A, B, C, and D) smaller than 0.1 (Song et al. 2001). The delays depend on both the refractor depth and velocity above it. The anisotropic strength can be defined as

$$
\Delta \mathrm{N}=\frac{\mathrm{V}_{\text {max }}-\mathrm{V}_{\text {min }}}{\mathrm{V}_{\text {max }}+\mathrm{V}_{\text {min }}} \times 200 \%
$$

where $\mathrm{V}_{\max }$ and $\mathrm{V}_{\text {min }}$ are the maximum and minimum propagation velocities, respectively. The set of linear Eq. (1) can be expressed in matrix form as $\mathbf{d}=\mathbf{A m}$ ( $\mathbf{m}$ - model vector, $\mathbf{d}$ - data vector) and solved using, for example, damped least squares (DLS) inversion for $\mathrm{a}_{\mathrm{i}}, \mathrm{b}_{\mathrm{j}}, \mathrm{S}_{0}$ and anisotropic parameters. For crustal anisotropic studies this method has been successfully used by Růžek et al. (2003) and Środa (2006). However, this method does not impose any smoothing constraints on the time delays at neighboring locations and requires high fold recordings. Due to the initial TAIGER experimental design for 2D seismic sections, some of the receivers recorded only one or two shots. If we include the receiver corrections $\left(b_{j}\right)$ in the inversion, the results might show low RMS residuals but unrealistic delay times and anisotropic parameters as well. Therefore, we decided to invert for the shot corrections $\left(a_{i}\right)$ only.

In order to verify if the anisotropic model is necessary to explain the data and to evaluate the significance of adding new model parameters, the inversion was performed in two variants: for isotropic and for anisotropic velocity. The uncertainties of the obtained parameters were calculated using the bootstrap method (Efron 1979). However, these uncertainties mainly reflect the random data errors effect and cannot be used to evaluate the inaccuracy resulting from assuming a simple model that may not be able to adequately image the inhomogeneous structure. This factor is hard to estimate reliably and therefore the actual errors are likely to be larger. The Backus (1965) formula is valid for near-horizontal rays in a weakly anisotropic medium and is not constrained to any particular symmetry. However, the only parameters that can be obtained from subhorizontal ray paths and from the measurements confined to a horizontal plane are the mean velocity, anisotropy, and $\theta_{\text {MAX }}$. Therefore, it is realistic to assume a model with the simplest anisotropic symmetry for the medium - transverse isotropy (TI) with a horizontal symmetry axis, which can be uniquely defined using these parameters. Such approach is justified by the fact that layered or fractured rocks often exhibit transversal isotropy. It should be noted that a study based on the described method could hardly discriminate between horizontal and (more likely) tilted axis TI medium. The observed magnitude of the anisotropy depends both on the rock properties and a symmetry axis dip, so they can- not be determined uniquely. Another approximation is due to the representation of a large, geologically complex crust fragment using constant parameters for the whole area.

\section{RESULTS}

Three inversion variants, assuming isotropy (ISO), $2 \theta$ anisotropy (ANI2 $\theta$ ) and $2 \theta+4 \theta$ anisotropy (ANI2 $\theta, 4 \theta$ ), were performed with different offset ranges to examine the robustness and reliability of the anisotropic inversion results (Fig. 4). ANI2 $\theta$ solves only the $2 \theta$ terms (A and B) of the elastic parameters of Eq. (1) instead of solving the $2 \theta$ and $4 \theta$ terms (A, B, C, and D) of the equation. The coefficients $\mathrm{C}$ and $\mathrm{D}$ are generally much smaller than $\mathrm{A}$ and $\mathrm{B}$, therefore most studies will ignore the $4 \theta$ terms to solve for fewer unknowns in inversion. However, here we inverted using both ANI2 $\theta$ and ANI2 $\theta, 4 \theta$ variants to test the stability of those coefficients. This inverse problem is linear with respect to the shot corrections ( 8 parameters for the dataset from shots) and with respect to the parameters defining isotropy ( 1 parameter), ANI2 $\theta$ (3 parameters) and ANI2 $\theta$, $4 \theta$ (5 parameters). The inversion results are summarized in Table 1.

In the inversion of the arrivals from 30 to $150 \mathrm{~km}$ offset results the final RMS residuals are 0.281 and $0.275 \mathrm{~s}$ for the ANI2 $\theta$ and ANI2 $\theta, 4 \theta$ models, respectively. They are significantly smaller than the residual for the isotropic model, $0.485 \mathrm{~s} \mathrm{(} 43 \%$ reduction compared to the isotropic model). The calculated anisotropic magnitude is $10.4 \%$ with $35.5^{\circ}$ fast Vp direction for the ANI2 $\theta$ model. The mean shot corrections of those three inversions are insignificant, which are $0.015 \mathrm{~s}, 0.002 \mathrm{~s}, 0.002 \mathrm{~s}$ for ISO, ANI2 $\theta$ and ANI $2 \theta, 4 \theta$ models, respectively. The coefficients of A and B are similar for both ANI2 $\theta$ variants and ANI2 $\theta, 4 \theta$ (Table 1 and Fig. 4). This justifies removing the station corrections from the inversions as they are expected to have similar magnitude. The mean velocity of the modeled anisotropic layer is $5.59 \mathrm{~km} \mathrm{~s}^{-1}$ for the ANI $2 \theta$ model.

In order to resolve more detailed anisotropic pattern variations in space we performed inversions of the arrivals in the near (30 to $60 \mathrm{~km}$ ) and far ( 60 to $150 \mathrm{~km}$ ) offsets and the arrivals central and north of the Central Range (Figs. 4 to 6 and Table 1). The strength of the anisotropy and the fast direction in the near and far offsets in the ANI2 $\theta$ model are 7.8 and $10.5 \%$ and 34.7 and $43.3^{\circ}$, respectively. The main sampling depths of the near and far offsets from the isotropic tomography ray paths of Kuo-Chen et al. (2012) are around $0-8$ and $8-15 \mathrm{~km}$, respectively (Fig. 5). The differences in the anisotropic strength and fast direction among the inversion results of the near, far offsets and $30-150 \mathrm{~km}$ offsets are within $3 \%$ and $8^{\circ}$ (Table 1 ). These slight differences show more detailed variations in anisotropy at different depths and the results of the $30-150 \mathrm{~km}$ offsets reveal the overall anisotropic pattern in the Central Range. Based on previous studies of local and teleseismic shear splitting 
Arrivals from 30 to $150 \mathrm{~km}$ offset
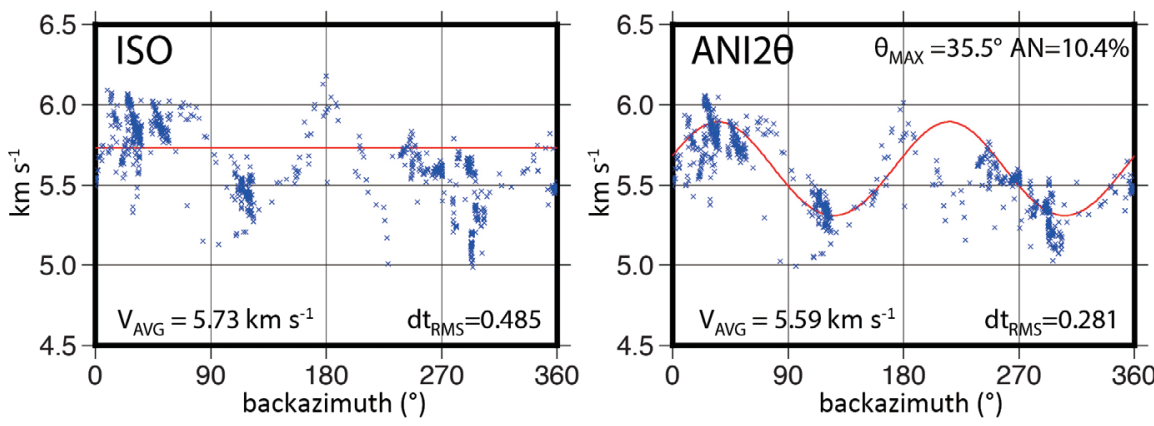

Arrivals from 30 to $60 \mathrm{~km}$ offset
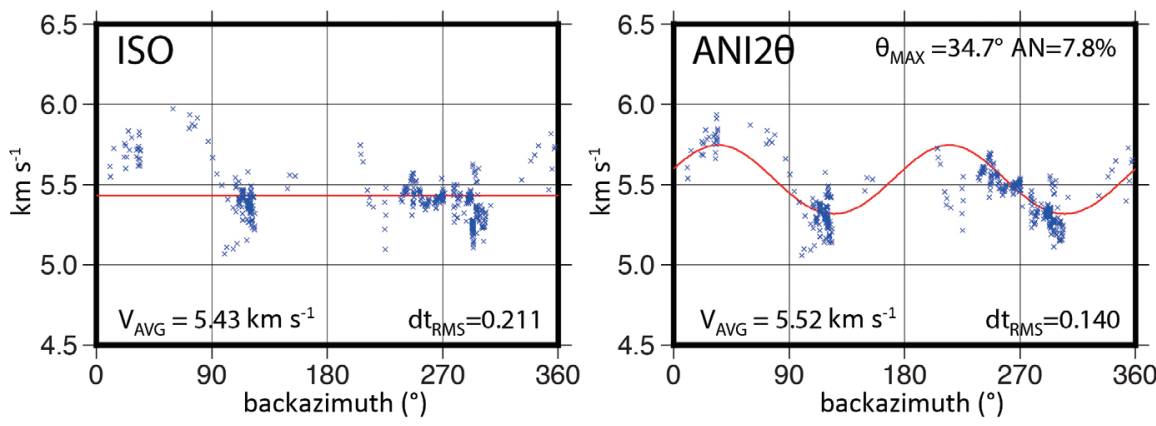

Arrivals from 60 to $150 \mathrm{~km}$ offset
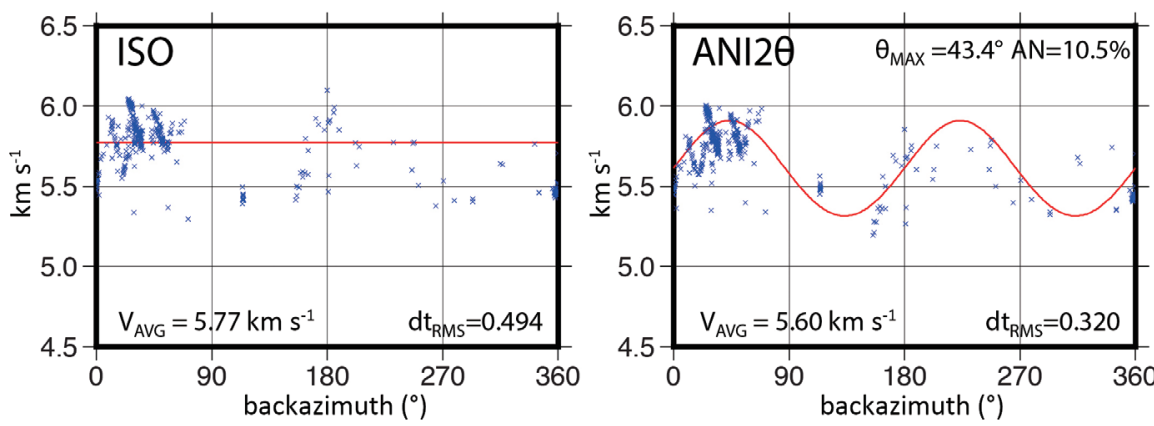
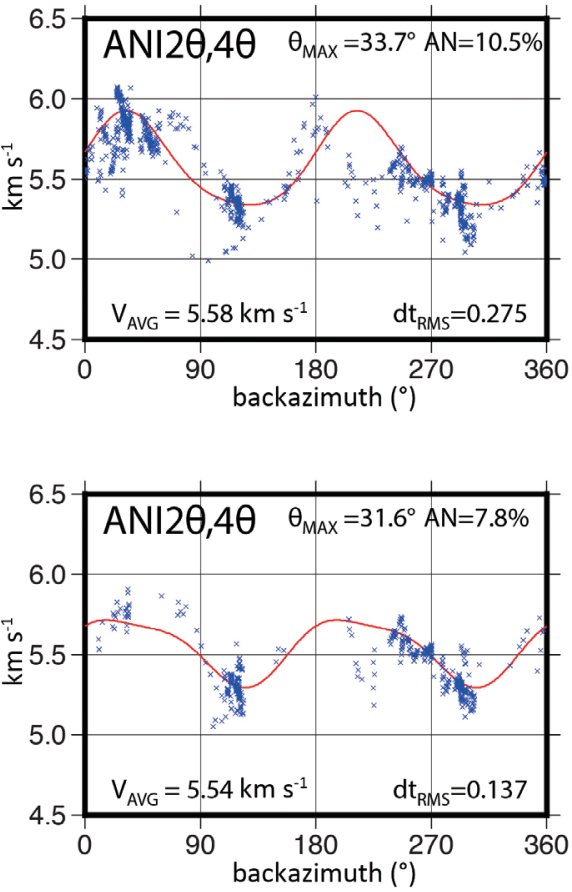

Fig. 4. Results of the isotropic, $2 \theta$ anisotropic, and $2 \theta+4 \theta$ anisotropic delay-time inversions with different offset ranges of arrivals. Blue dots: observed arrival. Red lines: inversion results.

Table 1. The Inversion Results.

\begin{tabular}{|c|c|c|c|c|c|c|c|c|c|c|c|c|c|}
\hline Method & $\begin{array}{c}\text { Mean } \\
\text { Vp km s } \text { s }^{-1}\end{array}$ & $\begin{array}{c}\text { Min. } \\
\text { Vp km s }^{-1}\end{array}$ & $\begin{array}{c}\text { Max. } \\
\text { Vp km s }^{-1}\end{array}$ & AN\% & $\boldsymbol{\theta}_{\mathrm{MAX}}$ & $\mathbf{A}$ & B & $\mathbf{C}$ & D & $\mathbf{N}_{\text {data }}$ & $\mathbf{N}_{\mathrm{par}}$ & DF & RMS dT sec \\
\hline \multicolumn{14}{|c|}{ Arrivals from 30 to $150 \mathrm{~km}$ offset } \\
\hline ISO & 5.73 & -- & -- & -- & -- & -- & -- & -- & -- & 1293 & 9 & 1284 & 0.485 \\
\hline ANI $2 \theta$ & 5.59 & 5.31 & 5.89 & 10.4 & 35.5 & -0.00303 & -0.00882 & -- & -- & 1293 & 11 & 1282 & 0.281 \\
\hline ANI $2 \theta, 4 \theta$ & 5.58 & 5.34 & 5.93 & 10.5 & 33.7 & -0.00356 & -0.00850 & 0.00067 & -0.00124 & 1293 & 13 & 1280 & 0.275 \\
\hline \multicolumn{14}{|c|}{ Arrivals from 30 to $60 \mathrm{~km}$ offset } \\
\hline ISO & 5.43 & -- & -- & -- & -- & -- & -- & -- & -- & 696 & 9 & 687 & 0.211 \\
\hline ANI $2 \theta$ & 5.52 & 5.32 & 5.75 & 7.8 & 34.7 & -0.00246 & -0.00657 & -- & -- & 696 & 11 & 685 & 0.140 \\
\hline ANI $2 \theta, 4 \theta$ & 5.54 & 5.29 & 5.72 & 7.8 & 31.6 & -0.00299 & -0.00593 & -0.00152 & 0.00076 & 696 & 13 & 683 & 0.137 \\
\hline \multicolumn{14}{|c|}{ Arrivals from 60 to $150 \mathrm{~km}$ offset } \\
\hline ISO & 5.77 & -- & -- & -- & -- & -- & -- & -- & -- & 597 & 9 & 588 & 0.494 \\
\hline ANI2 $\theta$ & 5.60 & 5.32 & 5.91 & 10.5 & 43.4 & -0.00054 & -0.00945 & -- & -- & 597 & 11 & 586 & 0.320 \\
\hline ANI $2 \theta, 4 \theta$ & 5.61 & 5.37 & 5.94 & 10.1 & 43.1 & -0.00052 & -0.00784 & 0.00101 & -0.00235 & 597 & 13 & 584 & 0.298 \\
\hline
\end{tabular}

Note: AN - strength of anisotropy, $\theta_{\text {MAX }}$ - fast direction of $V p, N_{\text {data }}$ - number of data points, $N_{\text {par }}$ - number of parameters, $D F$ - number of degrees of freedom. 


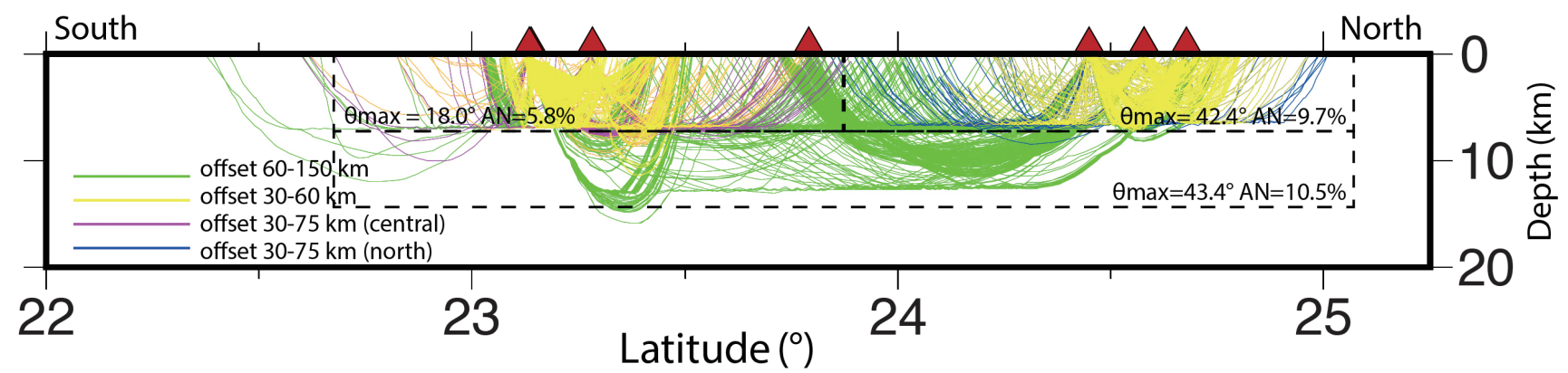

Fig. 5. Ray paths for different offset ranges. Three sub-regions (offset $30-75 \mathrm{~km}$ in the north, offset $30-75 \mathrm{~km}$ in the central, and offset 60 - $150 \mathrm{~km}$ ) separated by dashed lines. The ray paths calculated from isotropic tomography of Kuo-Chen et al. (2012). Red triangles: shot locations.

(a)

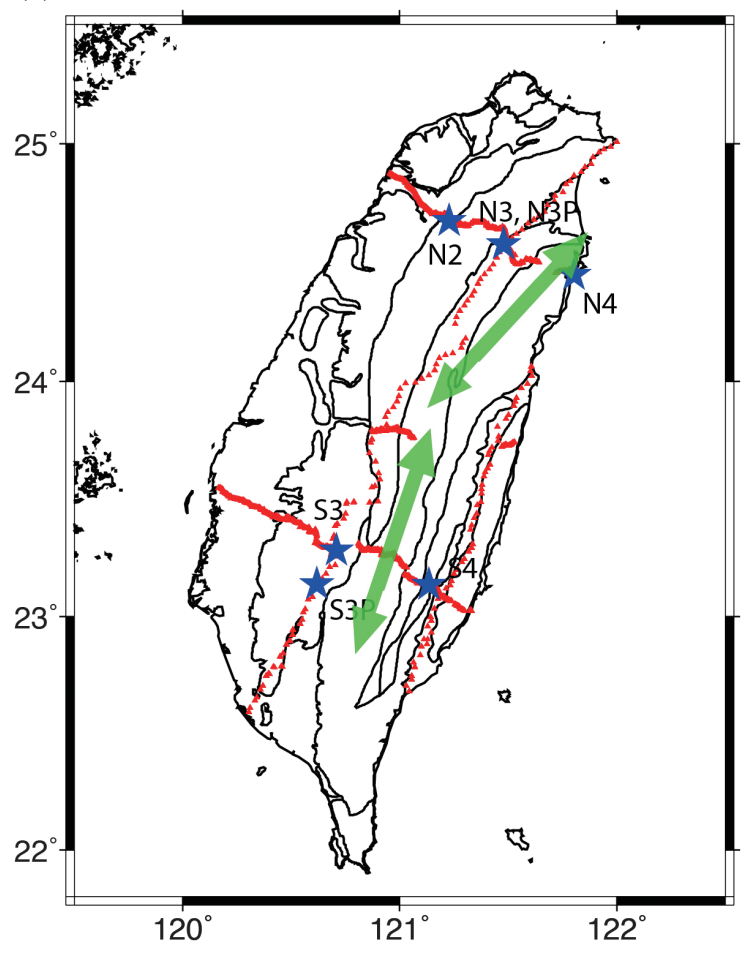

(b) N2, N3, N3P, N4 (offset: $30-75$ km)

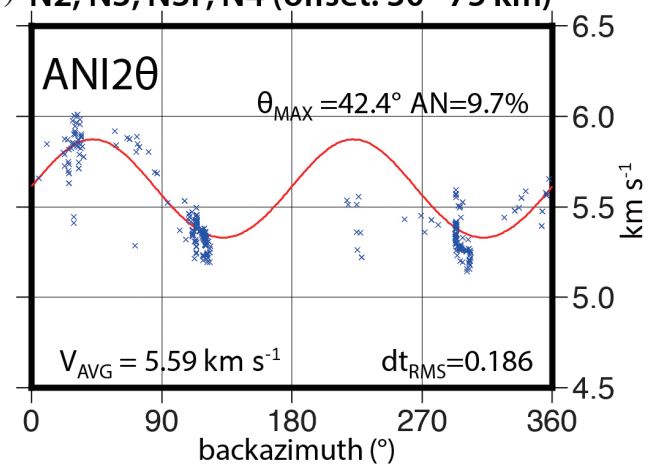

(c)

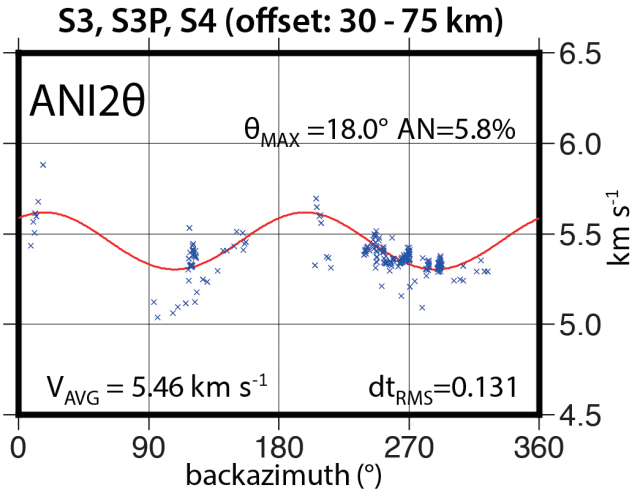

Fig. 6. Results from $2 \theta$ anisotropic inversions in northern and central mountain ranges. (a) $42.3^{\circ}$ and $18.0^{\circ}$ of the fast directions in northern and central ranges, respectively. Green arrows: fast directions. (b) The $2 \theta$ anisotropic inversions of N2, N3, N3P, and N4 shots within $30-75 \mathrm{~km}$ offsets in northern mountain ranges. (c) The $2 \theta$ anisotropic inversions of S3, S3P, and S4 shots within $30-75 \mathrm{~km}$ offsets in central mountain ranges. Blue dots: observed arrivals. Red lines: inversion results.

(Kuo et al. 1994; Kuo-Chen et al. 2009), the fast directions turn from NNW in the south to almost EW at the northern tip of the Central Range. We conducted separate inversions for the northern and central mountain ranges for testing the observations from previous studies. The results from the fast directions in the northern and central mountain belts coincide with the foliation pattern at the surface and the local and teleseismic shear wave splitting measurements as well, which are 18.0 and $42.4^{\circ}$ in the central and northern mountains (Fig. 6). The anisotropic strength increases from 5.8\% in the central to $9.7 \%$ in the north.

\section{DISCUSSION AND CONCLUSION}

In the slate belts of the Central Range, rock measurements show the average $P$ wave velocities are $5.6-6.0 \mathrm{~km} \mathrm{~s}^{-1}$ (Wu et al. 2007) and the 3D tomographic model shows $5.5-5.8 \mathrm{~km} \mathrm{~s}^{-1}$ of $\mathrm{P}$ wave velocities in the upper crust $(0-15 \mathrm{~km})$ in the Hsuehshan and Central Ranges (Kuo-Chen et al. 2012). The data from the explosion experiments used in this study mainly sampled the upper crust $(0-15 \mathrm{~km})$ of the mountain belts according to the ray tracing results for those arrivals from the isotropic tomography (Kuo-Chen et 
al. 2012) and the average $P$ wave velocity is $\sim 5.68 \mathrm{~km} \mathrm{~s}^{-1}$ (Fig. 2c). If we consider the errors produced from lateral velocity variations in the inversions from the different variants of inversions we performed, for example, the average Vp of the ANISO2 $\theta$ models with different offsets (Fig. 4) and with different regions (Fig. 6), a range between 5.46 and $5.60 \mathrm{~km} \mathrm{~s}^{-1}$, which could represent the vertical and horizontal velocity variations and the contribution of those lateral and vertical velocity variations to the uncertainty of the anisotropy is within $2 \%$. Those studies all show that a simple homogeneous crustal model is a good approximation and assumption for the upper crust $(0-15 \mathrm{~km})$ for the inversions. In this study we were able to show that the azimuthal variations of the delay times are due mainly to the anisotropic effect and can be described using a transverse isotropic model with a horizontal symmetry axis (HTI).

Several anisotropic studies at different scales in the mountain belts, the foliation trends at the surface (Fig. 1), rock measurements (Wu et al. 2007), crustal P wave anisotropy (this study) and local and teleseismic shear wave splitting measurements (Rau et al. 2000; Huang et al. 2006; Chang et al. 2009; Kuo-Chen et al. 2009), all consistently show the NNE fast directions. This phenomenon can be explained by the WSW orientation of the main compressional axis between the Eurasian and Philippine Sea plates - perpendicular to the fast directions. On the other hand, the strength of the anisotropy beneath the slate belts of the Central Range is $8-20 \%$, as known from laboratory rock measurements (Wu et al. 2007) and the results from this study show the upper crustal anisotropy $(0-15 \mathrm{~km})$ in the mountain ranges is $8-10 \%$. The similarities between the anisotropic parameters from rock measurements and from this study reveal coherent deformation from the surface to the upper crust. The strength of the crustal anisotropy $(0-30 \mathrm{~km})$ from local shear wave splitting measurements of one seismic station in the southern Central Range varies from 0 to $6 \%$ (Chang et al. 2009), but these results only represent the local effect beneath the seismic stations. The crustal anisotropy obtained in this study shows the overall upper crustal anisotropic pattern in the mountain ranges. In addition, the upper crustal anisotropy could contribute significantly to the delay times observed by teleseismic shear wave splitting measurements. Assuming $3.3 \mathrm{~km} \mathrm{~s}^{-1} \mathrm{~S}$ wave velocity and $10.4 \%$ anisotropy for the average upper crust [by assuming the anisotropic strength of Vs similar to Vp, (Lloyd et al. 2009, 2010)], the delay time can reach $\sim 0.45 \mathrm{~s}$ for the upper crust $(15 \mathrm{~km})$. As the total delays from SKS splitting in Taiwan mountain ranges reaches $1-2 \mathrm{~s}$, the delay time contribution from the middle/lower crust and the upper mantle could be around $0.55-1.55 \mathrm{~s}$, which still shows high anisotropy at greater depths as well. Therefore, the anisotropic data analyses from lab measurements (Wu et al. 2007), Pg in the upper crust (this study), and teleseismic shear wave splitting (Rau et al. 2000; Huang et al. 2006;
Kuo-Chen et al. 2009), reveal the vertically coherent deformation in the Taiwan orogen.

Acknowledgments The TAIGER project was supported by the Continental Dynamics Program of the National Science Foundation (EAR0410227 and EAR1010645). H. K-C was also supported by the National Science Council (NSC1012116-M-008-023-MY3). The assistance in figure preparation from C. -Y. Chiu, the initial discussion with D. Okaya and the comments by V. Schulte-Pelkum, S. Chevrot and one anonymous Reviewer are greatly appreciated.

\section{REFERENCES}

Backus, G. E., 1965: Possible forms of seismic anisotropy of the uppermost mantle under oceans. J. Geophys. Res., 70, 3429-3439, doi: 10.1029/JZ070i014p03429. [Link]

Chai, B. H. T., 1972: Structure and tectonic evolution of Taiwan. Am. J. Sci., 272, 389-422, doi: 10.2475/ ajs.272.5.389. [Link]

Chang, E. T. Y., W. T. Liang, and Y. B. Tsai, 2009: Seismic shear wave splitting in upper crust characterized by Taiwan tectonic convergence. Geophys. J. Int., 177, 1256-1264, doi: 10.1111/j.1365-246X.2009.04110.x. [Link]

Ching, K. E., M. L. Hsieh, K. M. Johnson, K. H. Chen, R. J. Rau, and M. Yang, 2011: Modern vertical deformation rates and mountain building in Taiwan from precise leveling and continuous GPS observations, 2000-2008. J. Geophys. Res., 116, B08406, doi: 10.1029/2011JB008242. [Link]

Efron, B., 1979: Bootstrap methods: Another look at the jackknife. Ann. Stat., 7, 1-26.

Ho, C. S., 1988: An Introduction to the Geology of Taiwan Explanatory Text of the Geologic Map of Taiwan, Central Geological Survey, Taiwan, 192 pp.

Huang, B. S., W. G. Huang, W. T. Liang, R. J. Rau, and N. Hirata, 2006: Anisotropy beneath an active collision orogen of Taiwan: Results from across islands array observations. Geophys. Res. Lett., 33, L24302, doi: 10.1029/2006GL027844. [Link]

Kuo, B. Y., C. C. Chen, and T. C. Shin, 1994: Split S waveforms observed in northern Taiwan: Implications for crustal anisotropy. Geophys. Res. Lett., 21, 1491-1494, doi: 10.1029/94GL01254. [Link]

Kuo-Chen, H., F. T. Wu, D. Okaya, B. S. Huang, and W. T. Liang, 2009: SKS/SKKS splitting and Taiwan orogeny. Geophys. Res. Lett., 36, L12303, doi: 10.1029/2009GL038148. [Link]

Kuo-Chen, H., F. T. Wu, and S. W. Roecker, 2012: Threedimensional $\mathrm{P}$ velocity structures of the lithosphere beneath Taiwan from the analysis of TAIGER and related seismic data sets. J. Geophys. Res., 117, B06306, doi: 10.1029/2011JB009108. [Link] 
Lloyd, G. E., R. W. H. Butler, M. Casey, and D. Mainprice, 2009: Mica, deformation fabrics and the seismic properties of the continental crust. Earth Planet. Sci. Lett., 288, 320-328, doi: 10.1016/j.eps1.2009.09.035. [Link]

Lloyd, G. E., R. D. Law, and D. Mainprice, 2010: Predicting seismic properties from three-dimensional microstructures: A new look at an old quartzite. Geol. Soc. London Spec. Publ., 335, 603-622, doi: 10.1144/SP335.25. [Link]

Rau, R. J., W. T. Liang, H. Kao, and B. S. Huang, 2000: Shear wave anisotropy beneath the Taiwan orogen. Earth Planet. Sci. Lett., 177, 177-192, doi: 10.1016/ S0012-821X(00)00058-3. [Link]

Růžek, B., V. Vavryčuk, P. Hrubcová, J. Zedník, and the CELEBRATION Working Group, 2003: Crustal anisotropy in the Bohemian Massif, Czech Republic: Observations based on Central European Lithospheric Experiment Based on Refraction (CELEBRATION) 2000. J. Geophys. Res., 108, doi: 10.1029/2002JB002242. [Link]

Silver, P. G., 1996: Seismic anisotropy beneath the con- tinents: Probing the depths of geology. Annu. Rev. Earth Planet. Sci., 24, 385-432, doi: 10.1146/annurev. earth.24.1.385. [Link]

Środa, P., 2006: Seismic anisotropy of the upper crust in southeastern Poland-effect of the compressional deformation at the EEC margin: Results of CELEBRATION 2000 seismic data inversion. Geophys. Res. Lett., 33, L22302, doi: 10.1029/2006GL027701. [Link]

Song, L. P., M. Koch, K. Koch, and J. Schlittenhardt, 2001: Isotropic and anisotropic $P_{n}$ velocity inversion of regional earthquake traveltimes underneath Germany. Geophys. J. Int., 146, 795-800, doi: 10.1046/j.0956540x.2001.01495.x. [Link]

Willmore, P. L. and A. M. Bancroft, 1960: The time term approach to refraction seismology. Geophys. J. R. Astr. Soc., 3, 419-432, doi: 10.1111/j.1365-246X.1960. tb01715.x. [Link]

Wu, F. T., L. Lavier, UT, and US and Taiwan TAIGER TEAMS, 2007: Collision Tectonics of Taiwan and TAIGER Experiments, Eos Trans. AGU, 88, Fall Meet. Suppl., Abstract T51A-0321. 